\title{
Bragg polaritons in a ZnSe-based unfolded microcavity at elevated temperatures
}

Cite as: Appl. Phys. Lett. 108, 121105 (2016); https://doi.org/10.1063/1.4944717

Submitted: 17 December 2015 . Accepted: 09 March 2016. Published Online: 23 March 2016

K. Sebald, SK. S. Rahman, M. Cornelius, (D T. Kaya, J. Gutowski, T. Klein, A. Gust, D. Hommel, and S. Klembt
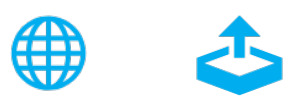

View Online

\section{ARTICLES YOU MAY BE INTERESTED IN}

Relaxation and emission of Bragg-mode and cavity-mode polaritons in a ZnO microcavity at room temperature

Applied Physics Letters 95, 121102 (2009); https://doi.org/10.1063/1.3232228

Perspective: Photonic flatbands

APL Photonics 3, 070901 (2018); https://doi.org/10.1063/1.5034365

Counter-directional polariton coupler

Applied Physics Letters 114, 061102 (2019); https://doi.org/10.1063/1.5067247

\section{Challenge us.}

What are your needs for periodic signal detection?

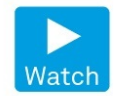

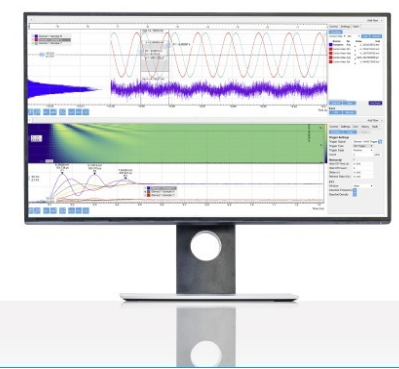

- Zurich

- Instruments 


\title{
Bragg polaritons in a ZnSe-based unfolded microcavity at elevated temperatures
}

\author{
K. Sebald, ${ }^{1}$ SK. S. Rahman, ${ }^{1}$ M. Cornelius, ${ }^{1}$ T. Kaya,${ }^{1}$ J. Gutowski, ${ }^{1}$ T. Klein, ${ }^{2}$ A. Gust,${ }^{2}$ \\ D. Hommel, 2, a) and S. Klembt ${ }^{3}$ \\ ${ }^{1}$ Semiconductor Optics, Institute of Solid State Physics, University of Bremen, 28334 Bremen, Germany \\ ${ }^{2}$ Semiconductor Epitaxy, Institute of Solid State Physics, University of Bremen, 28334 Bremen, Germany \\ ${ }^{3}$ Institut Néel, Université Grenoble Alpes and CNRS, B.P. 166, 38042 Grenoble, France
}

(Received 17 December 2015; accepted 9 March 2016; published online 23 March 2016)

\begin{abstract}
In this contribution, we present strong coupling of $\mathrm{ZnSe}$ quantum well excitons to Bragg modes resulting in the formation of Bragg polariton eigenstates, characterized by a small effective mass in comparison to a conventional microcavity. We observe an anticrossing of the excitonic and the photonic component in our sample being a clear signature for the strong-coupling regime. The anticrossing is investigated by changing the detuning between the excitonic components and the Bragg mode. We find anticrossings between the first Bragg mode and the heavy- as well as light-hole exciton, respectively, resulting in three polariton branches. The observed Bragg-polariton branches are in good agreement with theoretical calculations. The strong indication for the existence of strong coupling is traceable up to a temperature of $200 \mathrm{~K}$, with a Rabi-splitting energy of $24 \mathrm{meV}$ and $13 \mathrm{meV}$ for the Bragg mode with the heavy- and light-hole exciton, respectively. These findings demonstrate the advantages of this sample configuration for ZnSe-based devices for the strong coupling regime. (C) 2016 AIP Publishing LLC. [http://dx.doi.org/10.1063/1.4944717]
\end{abstract}

Strong light-matter interaction has attracted much interest in the last decade because of the possibility to study its fundamental aspects ${ }^{1,2}$ as well as to create optoelectronic devices, whose operation is based on the properties of cavity-exciton polaritons. ${ }^{3}$ The benefit of wide-bandgap semiconductorbased microcavities (MCs) is confirmed by the observation of strong coupling in $\mathrm{ZnO}$ and $\mathrm{GaN}$ MCs, resulting in polariton lasing at room temperature. ${ }^{4,5} \mathrm{ZnSe}$-based $\mathrm{MCs}$ are particularly well-suited for the investigation of cavity-exciton polaritons in semiconductors due to the high exciton oscillator strength and large exciton binding energy. We reported a promising Rabi-splitting energy of about $11 \mathrm{meV}$ per quantum well $(\mathrm{QW})^{6,7}$ as well as polariton lasing in micropillars. ${ }^{8}$ Since the Rabi-splitting energy increases with the number of quantum wells, an investigation of the strong-coupling regime in samples composed of $\mathrm{ZnSe}$ is even possible at elevated temperatures. In order to increase the Rabi-splitting energy in the latter MCs, more QWs must be used, which results in a thicker cavity. This hampers the increase of the Rabi-splitting energy due to its larger mode volume.

In recent years, Bragg polaritons have been discussed as an innovative tool for tailoring light-matter coupling. ${ }^{9,10}$ This type of unfolded MC sample is advantageous, because a large number of QWs can be positioned at the field maxima of the Bragg mode without increasing the mode volume. In this case, the light-matter interaction occurs between the QW excitons and the Bragg-mirror modes, leading to the creation of Bragg polariton eigenstates. These polaritons possess an extremely small effective mass in comparison to those in conventional MCs. ${ }^{11,12}$ Since the critical temperature for polariton condensation is inversely proportional to the polariton effective mass,

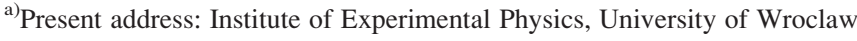
and Wroclaw Research Center EIT, 50204 Wroclaw, Poland.
}

such structures are promising for the realization of hightemperature polariton condensation. The strong light-matter coupling regime in a Bragg-polariton sample is indicated, as for a Fabry-Pérot MC, by an anticrossing of the excitonic resonance and the photonic mode. For conventional MCs, this effect has been reported previously. ${ }^{13-15}$ The first report on the realization of strong coupling for an unfolded $\mathrm{MC}$ concerned single InGaAs QWs embedded in a 30-period distributed Bragg reflector (DBR) stack with a Rabi-splitting energy of $9.3 \mathrm{meV}$. The strong-coupling regime was observed up to $100 \mathrm{~K} .^{9}$ For an alternative concept, 70-period GaAs/AlGaAs QWs were employed. From reflectivity measurements at $10 \mathrm{~K}$, the interaction strengths between light-hole $\left(\mathrm{X}_{\mathrm{lh}}^{1}\right)$ and heavyhole $\left(\mathrm{X}_{\mathrm{hh}}^{1}\right)$ excitons with the photons were reported to be $4.4 \mathrm{meV}$ and $6.2 \mathrm{meV}$, respectively. ${ }^{16}$ This Bragg-sample concept was used for all-organic one-dimensional photonic crystals as well, resulting in a branch splitting of about $75 \mathrm{meV} .{ }^{17}$ For the coupling of resonant Bragg gratings to $\mathrm{ZnO}$ bulk excitons, recent theoretical investigations have shown peculiar nonlinear phenomena, indicating that this system may be used to create slow-light-enhanced nonlinear propagation. ${ }^{12}$ Furthermore, Bragg-polariton samples were recently proposed as an innovative concept for designing an additional type of hyperbolic metamaterial, ${ }^{10}$ which is interesting for applications in, e.g., quantum lifetime engineering.

In this contribution, we report on the optical properties of a Bragg-polariton sample grown by molecular beam epitaxy (MBE). For this sample, all three $8 \mathrm{~nm}$ thick $\mathrm{ZnSe}$ QWs were embedded in the center of the high-index material made of $\mathrm{ZnMg}_{0.21} \mathrm{~S}_{0.23} \mathrm{Se}$ with a total thickness of $3 \lambda / 4(144 \mathrm{~nm})$. The QWs are separated by $5 \mathrm{~nm}$ of the quaternary material. The low-index material consists of a superlattice of 41 periods of $\mathrm{MgS}$ and $\mathrm{ZnCd}_{0.37} \mathrm{Se}$ with a total thickness of $\lambda / 4(44.7 \mathrm{~nm})$ (see Fig. 1(a)). This concept results in an effective overlap of 
the electric field of the first Bragg mode and the QWs as shown in the calculation in Fig. 1(b). Eight of these Braggpolariton layers were grown on four plain DBR pairs, where the latter ensures a precise in-situ control of the respective $\lambda / 4$ thickness. The high- and low-index materials possess a refractive index contrast on the order of $\Delta \mathrm{n}=0.4$, and the refractive index dispersions of both layers were taken from. ${ }^{18}$ In view of the sample's growth, this concept has the advantage of reducing the number of growth interfaces by a factor of 2.5 in comparison to conventional MCs for the strong-coupling regime, which we have previously presented. ${ }^{6,7}$ This simplification exhibits a positive impact on the crystal quality of the sample. Micro-reflectivity measurements with a white-light source (spot diameter $\sim 3 \mu \mathrm{m}$ ) were performed, and the experimental findings are compared to calculations using a transfer matrix (TM) method (CAMFR program ${ }^{19}$ ) as well as the coupled oscillator model for the Bragg mode, the heavy- and the lighthole exciton. ${ }^{20}$

In Fig. 1(c), an overview spectrum (spectral resolution $\mathrm{SP}=7 \mathrm{meV}$ ) of the Bragg sample is displayed for a temperature of $60 \mathrm{~K}$ in comparison to the TM-calculated reflectivity for such a sample without QWs. The stopband is centered at $2.75 \mathrm{eV}$, and the position of the first Bragg mode (BM) on the high-energy side can be identified at $2.823 \mathrm{eV}$ from the calculated spectrum. Hence, the resonance is detuned by approximately $12 \mathrm{meV}$ relative to the spectral position of $\mathrm{X}_{\mathrm{hh}}^{1}$ located at $2.811 \mathrm{eV}$. This almost resonant situation results in the detailed spectrum for reflectivity and PL, when highly spectrally resolved measurements were performed as shown in the inset $(\mathrm{SP}=0.1 \mathrm{meV})$. The reflectivity breaks up into five distinct minima. The minimum centered at $2.811 \mathrm{eV}$ originates from the $\mathrm{ZnSe} \mathrm{QW} \mathrm{X}_{\mathrm{hh}}^{1}$, while the minimum at $2.828 \mathrm{eV}$ can be attributed to the absorption of $\mathrm{X}_{\mathrm{lh}}^{1}$. This classification is underscored by the PL spectrum showing a dominant contribution of the $\mathrm{ZnSe} \mathrm{QW} \mathrm{X}_{\mathrm{hh}}^{1}$. The asymmetric shape of the emission is caused by a contribution of a trionic complex known to exist in $\mathrm{ZnSe} / \mathrm{ZnMgSSe}$ samples. ${ }^{21}$ Additionally, the PL has a shoulder on the low energy side at $2.79 \mathrm{eV}$ caused by increased transmission at the position of the reflectivity minimum. The first Bragg mode, identified at $2.823 \mathrm{eV}$ for the sample without QWs, is now split up into three reflectivity minima in the spectrum which can be identified at $2.793 \mathrm{eV}$,
$2.820 \mathrm{eV}$, and $2.834 \mathrm{eV}$. We name them the lower, middle, and upper Bragg-polariton (LBP, MBP, UBP) branches, respectively. In contrast to conventional MCs, Bragg-polariton samples show beside the polariton modes additional excitonic state contributions to the reflectivity spectrum. This is due to the QWs of the upper DBR layers, which are not efficiently coupled to the photonic modes, as discussed in Ref. 9. Additionally, the first excited state $\mathrm{X}_{\mathrm{hh}}^{2}$ can be identified as a less pronounced minimum in the spectra at $2.856 \mathrm{eV}$ (not shown).

We measured these reflectivity minima as a function of the local detection position on the sample at $4 \mathrm{~K}$ (Fig. 2(a)), where the average distance between those positions amounts to $300 \mu \mathrm{m}$. In doing so, we made use of the layer thickness gradient intrinsic to the MBE growth, which leads to a variation of the spectral position of any Bragg minimum, as shown in the figure for a higher Bragg mode (HBM, open circles). In contrast to the latter, the exciton-related absorption (stars) varies comparatively little with the thickness gradient. Hence, the detuning between the $X_{h h}^{1}$ and the first Bragg mode is changed from position to position allowing us to easily observe the anticrossing behavior of the three components of the first Bragg mode.

For the number of observable polariton branches, the exciton binding energy, the Rabi-coupling energy, and the energy separation $\Delta$ between $X_{h h}^{1}$ and $X_{\mathrm{lh}}^{1}$ are important. The Rabi-splitting energy corresponding to the exciton coupling strength amounts to $\hbar \Omega_{R}^{\mathrm{MC}}=19 \mathrm{meV}$ for three QWs in a microcavity based on $\mathrm{ZnSe}^{7}$ This value is comparable to the exciton binding energy of about $22 \mathrm{meV}$ for $8 \mathrm{~nm}$ thick $\mathrm{ZnSe}$ QWs in $\mathrm{ZnMgSSe}^{22}$ and its valence-band splitting of $18 \mathrm{meV}$. Hence, the anticrossing observed for varied detuning between the Bragg mode and excitonic emission in the reflectivity spectra results from the coupling of the Bragg mode with both contributions of $X_{h h}^{1}$ and $X_{\mathrm{lh}}^{1}$. Therefore, three eigenstates exist in the strong-coupling regime that are a combination of the Bragg-photon mode and the two excitonic states. They have already been labeled as LBP, MBP, and UBP, respectively. This attribution is underscored when the experimental findings are compared to the reflectivity minima extracted from the calculated reflectivity spectra using the TM method (see Fig. 2(b)). The positions of the $X_{h h}^{1}, X_{l h}^{1}$, and $X_{h h}^{2}$ are indicated by (a)

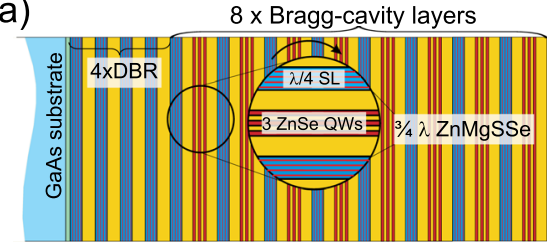

(b)

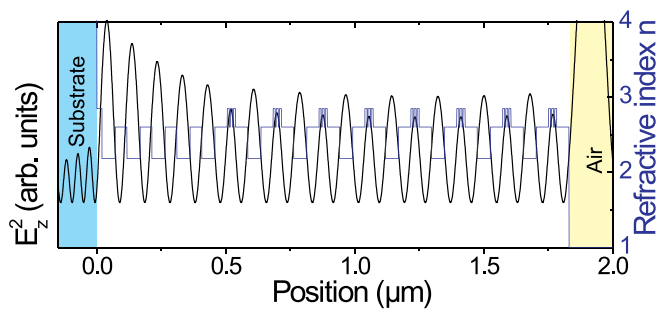

(c)

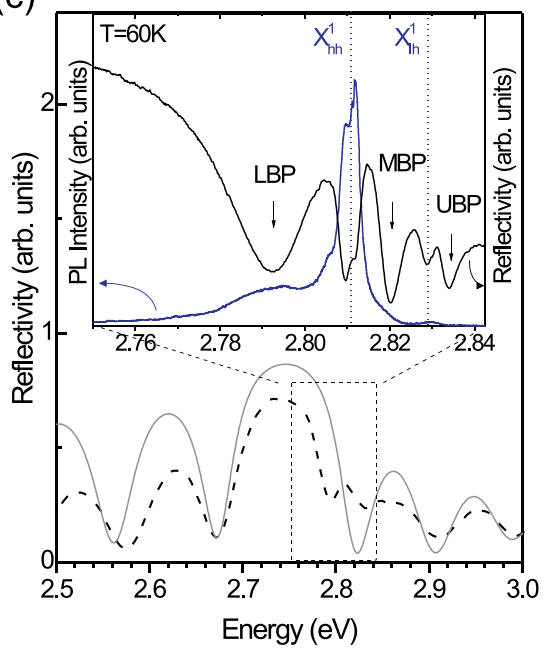

FIG. 1. (a) Sketch of the unfolded cavity structure. (b) Profile of the squared electric field distribution calculated for the energy of the first Bragg mode. The index of refraction is shown by the blue line. (c) Overview spectrum of the Bragg polariton sample at $\mathrm{T}=60 \mathrm{~K}$ (dashed line) compared to the calculated spectrum of a Bragg sample without QWs (solid line). The inset shows the high resolution reflectivity and PL spectra (excitation energy $3.03 \mathrm{eV}$ ) in the spectral region of the first Bragg mode. 
(a)

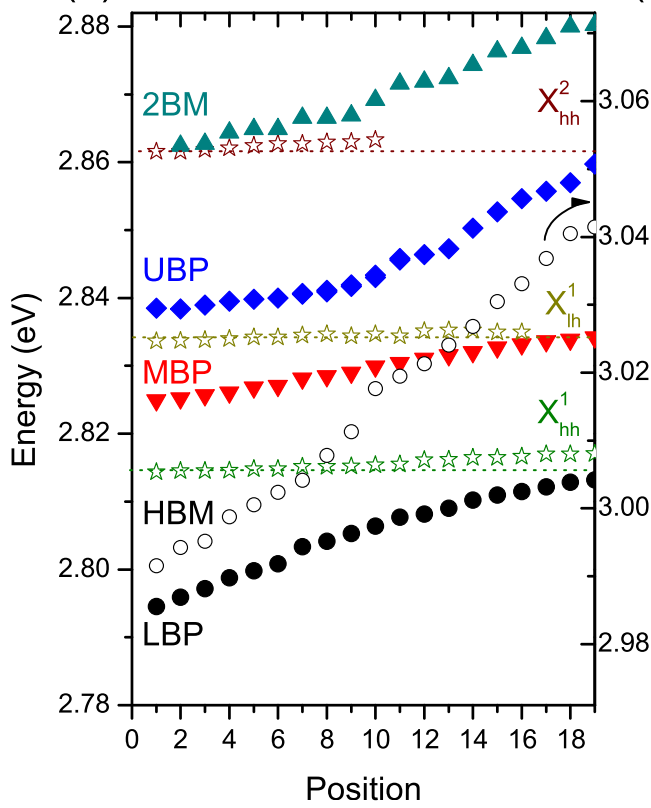

(b)

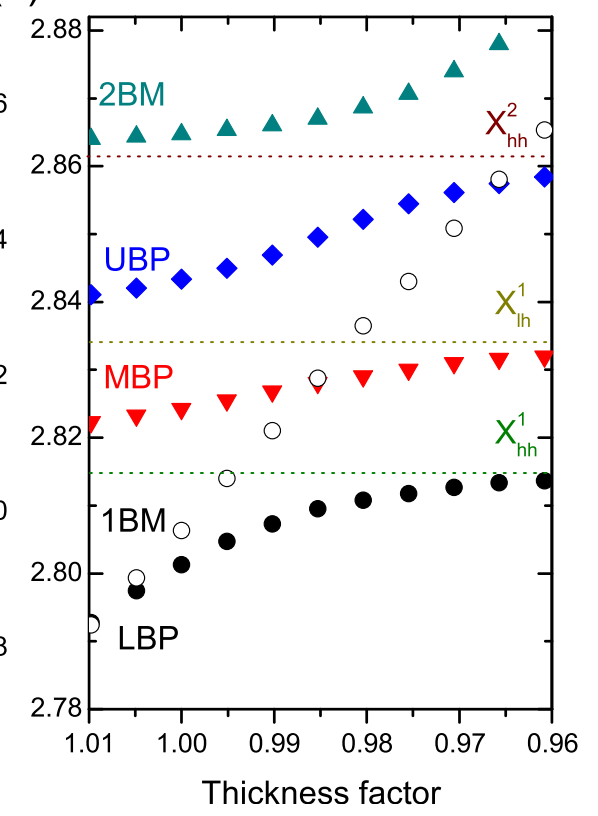

FIG. 2. (a) Experimental spectral reflectivity dip positions versus the local detection position number on the sample at $\mathrm{T}=4 \mathrm{~K}$. The numbers represent the positions on the sample from one edge to the other with a spacing of about $300 \mu \mathrm{m}$. The solid symbols represent the Bragg polariton modes. The stars correspond to the uncoupled excitonic states and the open circles to a higher Bragg mode (HBM). (b) Modeled spectral positions of Braggpolariton branches for different layer thicknesses normalized to the thickness for which the mode and $\mathrm{X}_{\mathrm{hh}}^{1}$ are in resonance (1.00). For comparison, the calculated first Bragg mode of a Bragg sample without QWs is plotted (open circles). horizontal lines. For the calculation, the $\mathrm{X}_{\mathrm{lh}}^{1} / \mathrm{X}_{\mathrm{hh}}^{1},\left(\mathrm{X}_{\mathrm{hh}}^{2} / \mathrm{X}_{\mathrm{hh}}^{1}\right)$ oscillator strength ratio was assumed to be $1 / 3,{ }^{23}$ (1/8 (Ref. 24)), and the thickness of the DBR layers was varied by the indicated factor. The 1.00 value was taken as the aspired norm thickness at which the Bragg mode and $X_{h h}^{1}$ are in resonance. The calculated evolution of the spectral positions of the reflectivity minima coincides quite well with the experimental findings (left side). It should be noted that we used a linear thickness gradient in the model for simplicity. However, the gradient on the 2-in. wafer becomes smaller towards the maximum thickness in the center, where we find the most negative detuning (in the direction of position 1 in Fig. 2(a)). For this reason, the model overestimates the thickness for small position numbers. Additionally, a particular spectral change of the excitonic emission for smaller thicknesses caused by a slight modification of the strain situation within the sample was not implemented in the calculation. This results in a more pronounced saturation of the spectral shift of the LBP for the calculation compared to the measurement. The three polariton branches show two anticrossings, one occurring between the Bragg mode and $\mathrm{X}_{\mathrm{hh}}^{1}$, and the second between the mode and $\mathrm{X}_{\mathrm{lh}}^{1}$. The interaction strengths between the excitons and the Bragg photons are $\hbar \Omega_{\mathrm{hh}}=(25 \pm 1) \mathrm{meV}$ and $\hbar \Omega_{\mathrm{lh}}=(13 \pm 1)$ $\mathrm{meV}$, respectively, as derived from the measurement at the resonance position of the Bragg mode and $\mathrm{X}_{\mathrm{hh}}^{1}$. These results indicate that although the spectral width of a Bragg mode $\left(\gamma_{\mathrm{BM}}=22 \mathrm{meV}\right)$ is large in comparison to a conventional MC cavity mode, the criterion for the strong coupling regime is still satisfied $\left(\hbar \Omega>\left(\left|\gamma_{\mathrm{X}}-\gamma_{\mathrm{BM}}\right|\right) / 2,{ }^{25}\right.$ with $\left.\gamma_{\mathrm{X}}=3 \mathrm{meV}\right)$.

The anticrossing can likewise be observed by detuning the excitonic components relative to the Bragg mode caused by a variation of the sample temperature. Fig. 3(a) depicts the reflectivity spectra in the vicinity of position 1 on the sample piece (Fig. 2(a)) in the temperature region between $40 \mathrm{~K}$ and $160 \mathrm{~K}$. They show the relative detuning of the reflectivity minima with respect to the energy position of $X_{h h}^{1}$ with the given temperatures taken as the zero vertical line. Besides reduced visibility of the excitonic components with increasing temperature, a clear spectral shift of the minima attributed to the LBP, MBP, and UBP can be seen with regards to the $X_{h h}^{1}$ position. This evolution is shown in detail in Fig. 3(b), where the relative detuning between the energy positions of the Bragg-polariton branches to those of $X_{h h}^{1}$ (again taken as 0) is plotted as a function of temperature (solid symbols). The measurement is shown in comparison to the calculated reflectivity minima based on the TM method (stars) where the spectral shift of excitonic components, their spectral broadening, and change of refractive index with temperature were taken into account. Additionally, the spectral shift of Bragg-polariton branches calculated by the three-oscillator model is plotted as a solid line (parameters were taken from the measurement). Furthermore, the changing position of the uncoupled Bragg mode due to the refractive index temperature dependence is shown by the dotted line. The measured Bragg polariton linewidth and the calculated excitonic linewidths (FWHM) are plotted as the shaded region. At this sample position, the anticrossing of the Bragg mode with $\mathrm{X}_{\mathrm{hh}}^{1}$ occurs at about $\mathrm{T}=130 \mathrm{~K}\left(\mathrm{FWHM}_{\text {calc }}=5 \mathrm{meV}\right)$, accompanied by a decrease of the LBP linewidth down to $4 \mathrm{meV}$. The anticrossing of the Bragg mode with $\mathrm{X}_{\mathrm{lh}}^{1}\left(\mathrm{FWHM}_{\mathrm{calc}}=8 \mathrm{meV}\right)$ is observed at $190 \mathrm{~K}$. The Rabi-splitting energies $\hbar \Omega_{\mathrm{hh}}$ and $\hbar \Omega_{\mathrm{lh}}$ deduced at the crossing point of $\mathrm{X}_{\mathrm{hh}}^{1}$ and the Bragg mode are approximately $(23 \pm 1) \mathrm{meV}$ and $(14 \pm 1) \mathrm{meV}$, respectively. The Rabi-splitting energy is proportional to the square root of the exciton oscillator strength $f$. Since $f\left(\mathrm{X}_{\mathrm{hh}}^{1}\right) / f\left(\mathrm{X}_{\mathrm{lh}}^{1}\right)=1 / 3$, one expects $\hbar \Omega_{\mathrm{hh}} / \hbar \Omega_{\mathrm{lh}}=1 / 1.75$, in good coincidence with the ratio of the experimentally determined values given above. For temperatures exceeding $200 \mathrm{~K}$, one observes a clear deviation between the results of the three-oscillator model and the measurement on one hand, and values from the TM method on the other. This is because for the fact that for the oscillator model, spectral broadening of the excitonic components was not taken into consideration. The reflectivity minima of the LBP and MBP start to approach $\mathrm{X}_{\mathrm{hh}}^{1}$ and $\mathrm{X}_{\mathrm{lh}}^{1}$, respectively, while the UBP matches with the uncoupled Bragg mode. Hence, the strong-coupling regime is broken for temperatures 

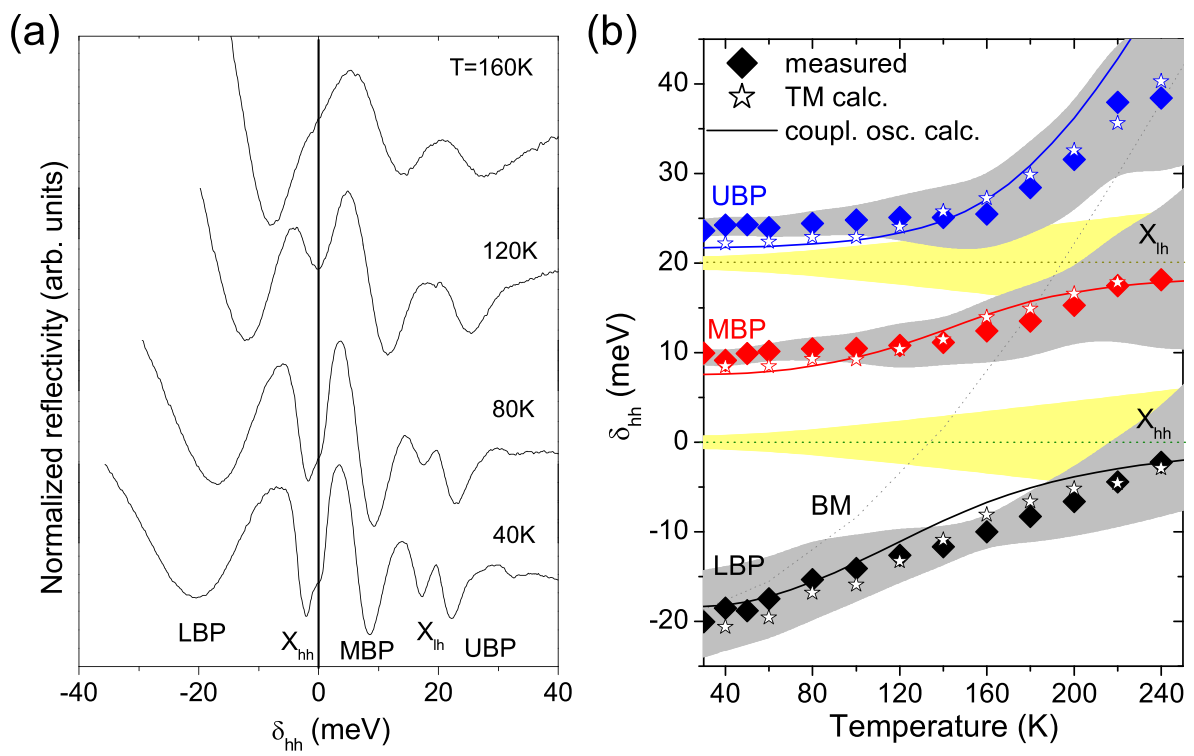

FIG. 3. (a) Reflectivity spectra for increasing temperatures, vertically shifted for clarity, and plotted versus relative energy position of the $X_{h h}^{1}$ taken as zero for respective temperatures. (b) Energy detuning of the Bragg polariton branches with the $X_{h h}^{1}$ energy taken as zero for respective temperatures. For comparison, the calculated reflectivity minima detuning is given when applying the TM method (stars) and the three-oscillator model (solid lines). The relative spectral shift of the uncoupled Bragg mode (BM) with regard to $X_{\text {hh }}^{1}$ is shown with the dotted line as well as the excitonic components. The measured Bragg polariton and the calculated excitonic linewidths are depicted as shaded areas.

above $200 \mathrm{~K}$ due to spectral broadening of the excitonic components.

These results show that a remarkably large Rabisplitting energy can be realized even with eight Braggpolariton stacks. This interaction strength is comparable to a complete MC structure consisting of an 18-fold bottom DBR, a $\lambda$ cavity, and a 15-pair top DBR, for which a Rabisplitting energy of $19 \mathrm{meV}$ was reported at $70 \mathrm{~K}{ }^{7}$ Hence, the smaller effective Bragg polariton mass relative to that in a conventional MC might take effect in the larger branch splitting and increase of temperature stability. As additional TM calculations have indicated, a further simplification of the sample setup would be possible by implementing only one QW at the antinode of the high-index material layer without a real reduction of the interaction strength. Additionally, the calculations show that the splitting energy between the LBP and UBP at $130 \mathrm{~K}$ can be raised from $36 \mathrm{meV}$ to $60 \mathrm{meV}$ by doubling the number of Bragg polariton stacks. The utilization of a threefold number of Bragg pairs providing an interaction strength of $75 \mathrm{meV}$ would make a ZnSe-based Braggpolariton sample comparable to organic Bragg structures, ${ }^{17}$ which are known for their large interaction strengths. These results are extremely encouraging with respect to the rapidly evolving field of nontrivial topological polariton effects. ${ }^{26-28}$ A recent work of Sedov et al. used a GaN/AlGaN structure for numerical calculations, with which Bragg polaritons were proposed to create a hyperbolic metamaterial. ${ }^{10}$ Our present results on a $\mathrm{ZnSe}$-based structure come quite close to benchmark values with the additional benefit of a high epitaxial quality.

In conclusion, we have shown strong coupling in an unfolded Bragg cavity with three ZnSe quantum wells embedded in each segment, repeated eight times. The interaction of the first Bragg mode with the exciton heavy-hole and light-hole states results in the formation of three polariton branches with an interaction strength of about $24 \mathrm{meV}$ and $13 \mathrm{meV}$, respectively. The anticrossing was analyzed by changing the detuning between the exciton components and the Bragg mode. The experimental findings are in excellent agreement with theoretical calculations. Although the Bragg modes possess a large spectral width and the growth process is simplified in comparison to a conventional microcavity, the interaction strength and temperature stability in the strong coupling regime are improved. This enables an additional class of II-VI based devices for strong coupling to be created.

We thank J. Dühn for his support in the oscillator model calculation. We gratefully acknowledge financial support from the Deutsche Forschungsgemeinschaft (Nos. Se 1846/ 1-2 and Ho 1388/33-2).

${ }^{1}$ J. Kasprzak, M. Richard, S. Kundermann, A. Baas, P. Jeambrun, J. M. J. Keeling, F. M. Marchetti, M. H. Szymańska, R. André, J. L. Staehli et al., Nature 443, 409 (2006).

${ }^{2}$ A. Amo, J. Lefrére, S. Pigeon, C. Adrados, C. Ciuti, I. Carusotto, R. Houdré, E. Giacobino, and A. Bramati, Nat. Phys. 5, 805 (2009).

${ }^{3}$ C. Schneider, A. Rahimi-Iman, N. Y. Kim, J. Fischer, I. G. Savenko, M. Amthor, M. Lermer, A. Wolf, L. Worschech, V. D. Kulakovskii et al., Nature 497, 348 (2013).

${ }^{4}$ G. Christmann, R. Butté, E. Feltin, J.-F. Carlin, and N. Grandjean, Appl. Phys. Lett. 93, 051102 (2008).

${ }^{5}$ F. Li, L. Orosz, O. Kamoun, S. Bouchoule, C. Brimont, P. Disseix, T. Guillet, X. Lafosse, M. Leroux, J. Leymarie et al.. Appl. Phys. Lett. 102, 191118 (2013).

${ }^{6}$ K. Sebald, A. Trichet, M. Richard, L. S. Dang, M. Seyfried, S. Klembt, C. Kruse, and D. Hommel, Eur. Phys. J. B 84, 381 (2011).

${ }^{7}$ K. Sebald, M. Seyfried, S. Klembt, S. Bley, A. Rosenauer, D. Hommel, and C. Kruse, Appl. Phys. Lett. 100, 161104 (2012).

${ }^{8}$ T. Klein, S. Klembt, E. Durupt, C. Kruse, D. Hommel, and M. Richard, Appl. Phys. Lett. 107, 071101 (2015).

${ }^{9}$ A. Askitopoulos, L. Mouchliadis, I. Iorsh, G. Christmann, J. J. Baumberg, M. A. Kaliteevski, Z. Hatzopoulos, and P. G. Savvidis, Phys. Rev. Lett. 106, 076401 (2011).

${ }^{10}$ E. S. Sedov, I. V. Iorsh, S. M. Arakelian, A. P. Alodjants, and A. Kavokin, Phys. Rev. Lett. 114, 237402 (2015).

${ }^{11}$ E. M. Kessler, M. Grochol, and C. Piermarocchi, Phys. Rev. B 77, 085306 (2008).

${ }^{12}$ F. Biancalana, L. Mouchliadis, C. Creatore, S. Osborne, and W. Langbein, Phys. Rev. B 80, 121306(R) (2009).

${ }^{13}$ M. Richard, R. Romestain, R. André, and L. S. Dang, Appl. Phys. Lett. 86, 071916 (2005).

${ }^{14}$ S. Faure, C. Brimont, T. Guillet, T. Bretagnon, B. Gil, F. Médard, D. Lagarde, P. Disseix, J. Leymarie, J. Zúñiga Pérez et al., Appl. Phys. Lett. 95, 121102 (2009). 
${ }^{15}$ E. Trichas, N. T. Pelekanos, E. Iliopoulos, E. Monroy, K. Tsagaraki, A. Kostopoulos, and P. G. Savvidis, Appl. Phys. Lett. 98, 221101 (2011).

${ }^{16}$ D. Goldberg, L. Deych, A. Lisyansky, Z. Shi, V. Menon, V. Tokranov, M. Yakimov, and S. Oktyabrsky, Nat. Photonics 3, 662 (2009).

${ }^{17}$ G. H. Lodden and R. J. Holmes, Phys. Rev. Lett. 109, 096401 (2012).

${ }^{18}$ S. Klembt, H. Dartsch, M. Anastasescu, M. Gartner, and C. Kruse, Appl. Phys. Lett. 99, 151101 (2011).

${ }^{19} \mathrm{P}$. Bienstmann, Rigorous and Efficient Modeling of Wavelength Scale Photonic Components (Universiteit Gent, Belgium, 2001).

${ }^{20}$ J. R. Jensen, P. Borri, W. Langbein, and J. M. Hvam, Appl. Phys. Lett. 76, 3262 (2000).

${ }^{21}$ G. V. Astakhov, V. P. Kochereshko, D. R. Yakovlev, W. Ossau, J. Nürnberger, W. Faschinger, and G. Landwehr, Phys. Rev. B 62, 10345 (2000).
${ }^{22}$ T. Miyajima, F. P. Logue, J. F. Donegan, J. Hegarty, H. Okuyama, A. Ishibashi, and Y. Mori, Appl. Phys. Lett. 66, 180 (1995).

${ }^{23}$ H. Kumano, H. Nashiki, I. Suemune, M. Arita, T. Obinata, H. Suzuki, K. Uesugi, and J. Nakahara, Phys. Rev. B 55, 4449 (1997).

${ }^{24}$ P. Y. Yu and M. Cardona, Fundamentals of Semiconductors (Springer, Berlin, 1996).

${ }^{25}$ V. Savona, C. Piermarocchi, A. Quattropani, P. Schwendimann, and F. Tassone, Phase Trans. 68, 169 (1999).

${ }^{26}$ T. Karzig, C.-E. Bardyn, N. H. Lindner, and G. Refael, Phys. Rev. X 5, 031001 (2015).

${ }^{27}$ C.-E. Bardyn, T. Karzig, G. Refael, and T. C. H. Liew, Phys. Rev. B 91, 161413 (2015).

${ }^{28}$ A. V. Nalitov, D. D. Solnyshkov, and G. Malpuech, Phys. Rev. Lett. 114, 116401 (2015). 